\title{
Innovations in MD-only physician-scientist training: experiences from the Burroughs Wellcome Fund physician-scientist institutional award initiative
}

\author{
Allison T. McElvaine, ${ }^{1}$ Jacqueline A. Hawkins-Salsbury, ${ }^{2}$ Vineet M. Arora, ${ }^{3}$ Mark T. Gladwin, ${ }^{4}$ James R. Goldenring, ${ }^{5}$ \\ David P. Huston, ${ }^{6}$ Deborah Krakow, ${ }^{7}$ Kyu Rhee, ${ }^{8}$ Julian Solway, ${ }^{3,9}$ Richard A. Steinman, ${ }^{4}$ Dwight A. Towler, ${ }^{10}$ Paul J. Utz, ${ }^{11}$ \\ Wayne M. Yokoyama, ${ }^{2,12}$ Rolly L. Simpson, ${ }^{13}$ Louis J. Muglia, ${ }^{13}$ Sallie R. Permar, ${ }^{14}$ and Rasheed A. Cbadegesin ${ }^{1,15}$
}

'Office of Physician-Scientist Development, Duke University School of Medicine, Durham, North Carolina, USA. Division of Physician-Scientists, Washington University School of Medicine, St. Louis, Missouri, USA. ${ }^{3}$ Department of Medicine, University of Chicago Pritzker School of Medicine, Chicago, Illinois, USA. ${ }^{4}$ Department of Medicine, University of Pittsburgh School of Medicine, Pittsburgh, Pennsylvania,

USA. ${ }^{5}$ Section of Surgical Sciences, Vanderbilt University School of Medicine, Nashville, Tennessee, USA. ${ }^{6}$ Clinical Science and Translational Research Institute, Texas A\&M University College of Medicine, Bryan, Texas, USA. 'Department of Obstetrics \& Gynecology, David Geffen School of Medicine, UCLA, Los Angeles, California, USA. ${ }^{8}$ Department of Medicine, Weill Cornell Medicine, New York, New York, USA. IInstitute for Translational Medicine, University of Chicago, Chicago, Illinois, USA. ${ }^{10} \mathrm{Department}$ of Internal Medicine, University of Texas Southwestern Medical Center, Dallas, Texas, USA. "Department of Medicine, Division of Immunology and Rheumatology, and the Institute for Immunity, Transplantation and Infection, Stanford University School of Medicine, Stanford, California, USA. ${ }^{22 D e p a r t m e n t ~ o f ~}$ Medicine, Washington University School of Medicine, St. Louis, Missouri, USA. ${ }^{3}$ Burroughs Wellcome Fund, Research Triangle Park, Durham, North Carolina, USA. ${ }^{14}$ Department of Pediatrics, Weill Cornell Medicine/NewYork-Presbyterian Hospital, New York, New York, USA. ${ }^{15}$ Department of Pediatrics, Duke University School of Medicine, Durham, North Carolina, USA.

\section{Introduction}

In the first half of the 20th century, while the value of translating scientific discovery to clinical care was clearly established, the pace of innovation was resulting in increased specialization in both medicine and science - and increased duration of their respective training paths. In the 1950s and 1960s, American doctors were drafted to serve in the Korean and Vietnam Wars (1). In 1953, the National Institutes of Health (NIH) started its Associate Training Program, which selected physicians who applied to the US Public Health Service Commissioned Corps to serve as clinical associates at the NIH (2). Associates were allowed to pursue mentored research training at the NIH and a unique opportunity for physician-scientist training emerged (3). In 1956, the NIH launched its Experimental Training Program to provide medical students with opportunities for summer research experiences or additional years of training focused on research (4). Such programs have produced leading scientists, including several Nobel Prize laureates, members of the National Academy of Sciences, and directors and institute directors at the NIH (1).

Individual medical schools began offering combined $\mathrm{MD}-\mathrm{PhD}$ training in the 1950s, and in 1964, the Nation-
Conflict of interest: VMA reports spouse stock ownership in Acadia Pharmaceuticals. MTC receives research support from Globin Solutions, Inc., the Institute for Transfusion Medicine, and the Hemophilia Center of Western Pennsylvania; is coinventor on patents and patent applications directed to the use of recombinant neuroglobin and heme-based molecules as antidotes for CO poisoning (61/187,527; 9,114,109; 14/776,363; 15/313,231; 62/525,909), which have been licensed by Globin Solutions, Inc.; is a shareholder, advisor, and director in Globin Solutions, Inc.; is coinventor on patents directed to the use of nitrite salts in cardiovascular diseases filed as international application numbers PCT/US2004/022232 (published as WO 2005/004884) and PCT/US2004/021985 (published as WO 2005/007173); and US 10/563,683 and US 12/748,184, which were previously licensed to United Therapeutics and are now licensed to Globin Solutions and Hope Pharmaceuticals; is a principal investigator in a research collaboration with Bayer Pharmaceuticals to evaluate riociguat as a treatment for patients with sickle cell disease is serving or has served as a scientific consultant for Actelion, Pfizer, Bayer Healthcare, Fulcrum, and Novartis; previously served as a consultant for Acceleron Pharma, Inc., Sujana Biotech, Epizyme, Inc., Catalyst Biosciences, Inc., Complexa, United Therapeutics, and Modus Therapeutics; and is on Bayer HealthCare LLC's Heart and Vascular Disease Research Advisory Board. DPH holds patents nos. 5,976,821 and 6,395,274 (Anti-Thioredoxin Monoclonal Antibodies) licensed to Genetics Institute (1995), Invitrogen Corp. (1995), Santa Cruz Biotechnology, Inc. (2000); and US patent application nos. 16/348,829 and 16/348,829. JS has interest in PulmOne Advanced Medical Diagnostics, Ltd, Israel, and is inventor on two patents pending (PCT/US20/26371 submitted to USPTO April 2, 2020 and PCT/ US20/26383 submitted to USPTO April 2, 2020). SRP has sponsored research agreements with and consults for Merck and Moderna. RLS is Senior Program Officer at the Burroughs Wellcome Fund. LJM is President and CEO at the Burroughs Wellcome Fund

Copyright: ( 2021, American Society for Clinical Investigation.

Reference information: / Clin Invest. 2021;131(10):e149948. https://doi.org/10.1172/JCI149948. al Institute of General Medical Sciences (NIGMS) launched the Medical Scientist Training Program (MSTP) to facilitate $\mathrm{MD}-\mathrm{PhD}$ dual-degree training programs (4). MSTP graduates are well represented in academia (4); yet, they comprise a small pool of talent and the total number of physicians engaged in research in the United States continues to diminish. While 41,178 research doctorate degrees were conferred in science and engineering fields in the United States in 2016 (5), only about 600 dual-degree MD-PhD students are graduated annually (6). The much larger pool of MD-only graduates (approximately 20,000 annually) therefore represents untapped potential to substantially increase the number of physicians engaged in research. However, most US MD programs focus on clinical training without a research component.

Accordingly, the Burroughs Wellcome Fund (BWF) launched an initiative in 2017 to develop a new cadre of MD-only physician-scientists by addressing barriers particular to them. The BWF Physician-Scientist Training Initiative infused \$25 million in flexible Physician-Scientist Institutional Awards (PSIAs), granted to ten medical schools who proposed innovative approaches to MD-only physicianscientist development. For the purposes of this initiative, "physician-scientist" is defined as a medical student or physician (MD, DO, or equivalent) actively engaged in laboratory-based discovery science. 
The BWF identified several common barriers to physician-scientist training: (a) lack of mentoring/role models, (b) lack of structured research training/experience in discovery science, (c) financial pressures and debt, (d) limited administrative support/centralized structure for physician-scientist training pathways, (e) burden of clinical training/responsibilities, and (f) career-personal life balance (7). Based on institutional support, commitment to diversity, scalability, and longitudinal evaluation plan, BWF awarded PSIA grants to ten institutions: Duke University (8), Stanford University (9), University of Pittsburgh (10), University of Texas Southwestern Medical Center (11), Vanderbilt University (12), Texas A\&M University (13), UCLA (14), University of Chicago (15), Washington University (16), and Weill Cornell Medicine (17). The five-year, \$2.5 million grants support development, testing, evaluating, disseminating, and sustaining novel approaches that address the barriers to physician-scientist training, with the ultimate goal of developing a new generation of physician-scientists who advance biomedical discovery to improve human health.

\section{Description and administration of programs}

Summary and categories of institutional approaches. PSIA institutions have implemented their programs (Figure 1), featuring many common elements. Individual approaches include structured education/ training/professional development; funding opportunities (for research support, tuition, or personal life use); integrated research training programs; mentorship; and new degree and certificate programs. Many programs feature distinctive elements, including networking and community building, connections with regional experts and infrastructure, and leadership training. Collectively, PSIA programs serve medical students, residents, fellows, instructors, and early-career faculty - with some targeting a single vulnerable group and others addressing the entire pipeline.

Institutional support. Institutional support provides avenues for sustainability of effective approaches. All PSIA grantees indicated institutional matching funds and leveraging/consolidation of preexisting programs for more centralized institution- al approaches to supporting physician-scientist development. Institutional support includes funds provided by schools and health systems, Deans' offices, and departments; administrative (staff and faculty leadership) support; funding for specific programmatic activities; philanthropic gifts; and commitments to sustain support at the conclusion of the funding period.

Diversity, equity, and inclusion. PSIA grantees report dedication to serving diverse constituencies and addressing the systemic racism that has perpetuated underrepresentation and inequity. All programs are collecting participant demographic information. Most are also collecting information on disability and disadvantaged-background status. At this early time point, one-third of PSIA programs reported that less than $10 \%$ of their participants were from racial and ethnic populations underrepresented in medicine (URiM) backgrounds, one-third reported between $10 \%$ and $25 \%$, and the remainder reported between $26 \%$ and 50\% participants from URiM backgrounds. In two-thirds of programs, women made up $51 \%-75 \%$ of participants, and one-third reported that $25 \%-50 \%$ of their participants are female.

Approaches to enhance diversity, equity, and inclusion have included workshops and trainings, formal integration of diversity and inclusion leadership in program teams, and assessment of programmatic reach to URiM individuals. Because individuals from URiM backgrounds and women are poorly represented at senior faculty and leadership levels, diversity among potential mentors is limited. PSIA grantees report leveraging peer and "nearpeer" mentorship for enhanced diversity among role models, providing culturally aware mentor training, and investing in long-term strategies to increase diversity in the physician-scientist pipeline by focusing on programs and activities that provide early exposure to the career path.

\section{Program implementation}

All PSIA institutions established a new administrative office or other structure to coordinate programs, which included hiring staff and establishing leadership positions, securing mentors and research preceptors, raising matching funds, developing evaluation and tracking tools; and establishing effective communication strategies for recruitment and promotion.

Implementation barriers. Programs intending to offer preferred admission to next-stage training (i.e., residency or fellowship physician-scientist training programs [PSTPs]) at times discovered challenges with the match process that precluded a strategic transition. Furthermore, programs found that Graduate Medical Education policies or time and resource constraints might limit support of research-in-residency opportunities for patient-oriented discovery science. Practical challenges included limitations in accessibility and quality of institutional data on students, trainees, and faculty needed to identify the target audience for PSIA initiatives; as well as the substantial time required to organize infrastructure, hire staff, and promote acceptance of programs by participants.

Lessons learned. To develop the resilience required to simultaneously master medicine and science, camaraderie is key. PSIA grantees have established mentorship teams, peer networks, advanced career development content, and executive coaching to this end. Compensation for mentor effort offers an approach to incentivize participation. The provision of financial and human resources (e.g., technician support) facilitates difficult transitions between research and clinical training. Vertical integration across the physician-scientist community contributes to promoting program engagement, visibility, and retention across training and career transitions. Careful coordination and communication with administrators and regulatory and accreditation bodies - and across existing programs and infrastructure - is necessary both to promote buy-in and to navigate potential political sensitivities or territoriality.

Institutional changes. All ten PSIA grantees launched substantial organizational changes, with the development of new centralized divisions or offices that serve as "home" for physician-scientists; new associate dean or other leadership positions; and/or new curriculum, tracks, or Master's and certificate programs in medical school training, residency, or fellowship programs.

National-level changes. Efforts and early outcomes of PSIA programs and projects 


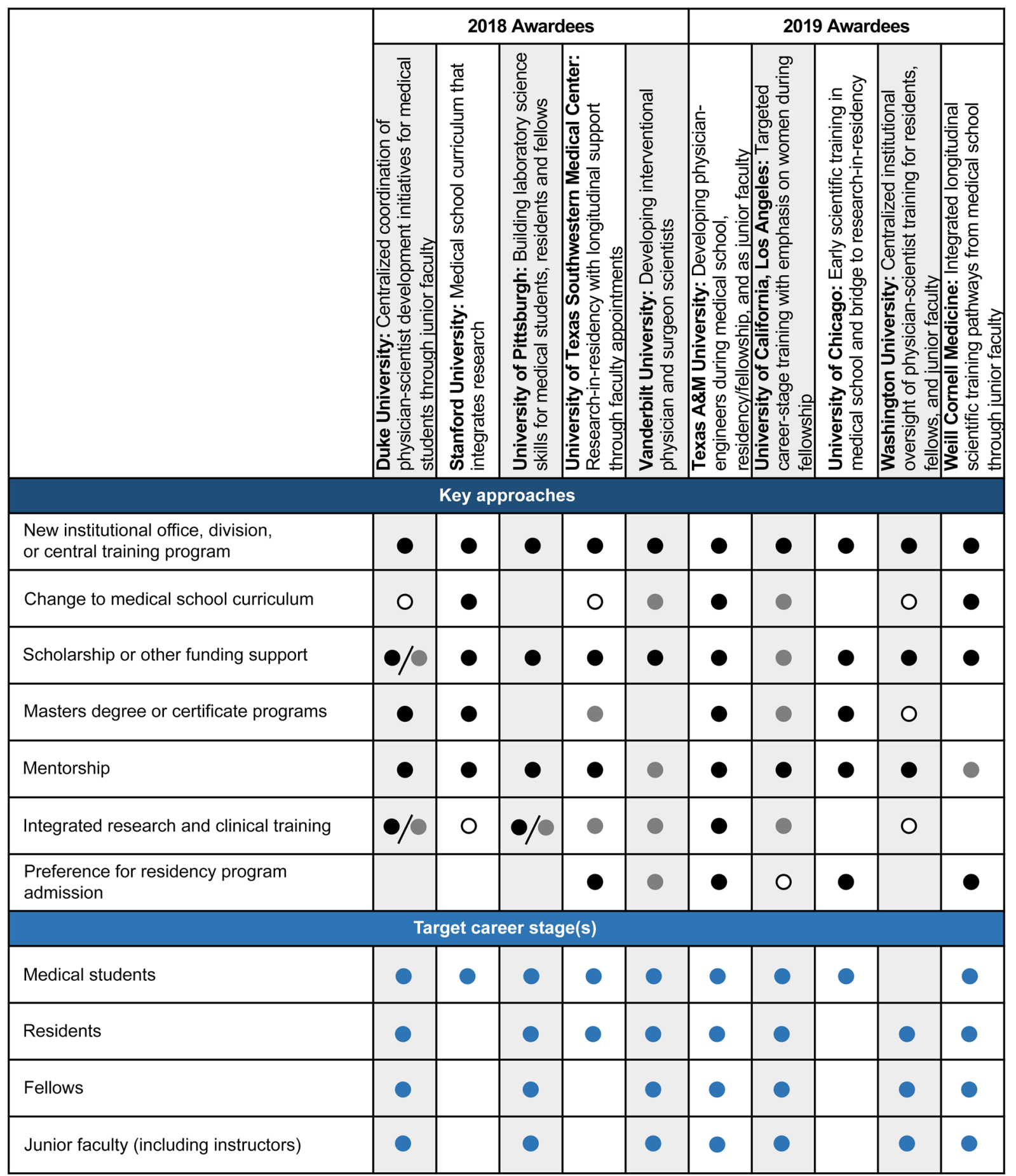

Figure 1. PSIA-funded programs, approaches, and career stage targets. PSIA institutions completed a survey to catalog and compare categorical key approaches across institutions as well as the targeted career stage by program. Key approaches were categorized and approaches newly launched with the PSIA are indicated with black-filled circles. Those that were preexisting at an awardee institution, but incorporated into the PSIA program, are indicated with gray-filled circles. Approaches that were preexisting and not incorporated into the PSIA program are indicated with open circles. Career stage targets by program are indicated with blue-filled circles.

have begun to influence the landscape of physician-scientist training at a national level. For example, a new nonprofit organization, the Physician Scientist Support Foundation, is working nationally to estab- lish an MD-only program that supports a gap year for research (18). Additionally, the first Association of American Medical Colleges Liaison Committee on Medical Education-approved integrated medicine and engineering curriculum has been established at a PSIA institution, providing a model for similar approaches. Buttressed by their organizational infrastructures, PSIA grantees have successfully compet- 
ed for a large share (7 of 21) of the NIH Stimulating Access to Research in Residency R38 pilot awards granted to date. In turn, several national medical boards have approved an R38 pathway for research in residency at multiple institutions.

Traditional metrics. PSIA grantees are tracking the outcomes of program participants to gauge success. All grantees intend to compare their program participants to MD-PhD students, baseline, or nonparticipants and to follow metrics of scholarly output (grant applications/success, publications/impact factors, presentations, honors and awards, patents/licenses) and career progression, as appropriate for the stage of intervention. Additionally, all programs are tracking attendance/ enrollment, matriculation, attrition, and demographics of participants. PSIA grantees also report plans to assess the level of engagement of participants, satisfaction with programs and mentors, and longterm career plans via survey instruments.

Nontraditional metrics. Individual PSIA grantees have identified various plans to track nontraditional metrics, including the following validated tools: resilience/grit scale, intrinsic/extrinsic motivation scale, career adaptabilities scale, burnout scale, and career/life satisfaction scale. Other unique metrics planned for evaluation include surveys to assess perceived selfefficacy in research and mentoring relationships; and a survey tool called Diagnostic Assessment of Research Training Strengths that will identify real and perceived barriers to MD-based research careers at different stages of training; better identify the personal training and mentoring needs of incoming trainees; and identify features of research training support that may differ between men, women, and/or individuals from URiM backgrounds.

Additional metrics will include resource utilization and impact at different career stages; curriculum evaluation; interdisciplinary faculty evaluation, trainee self-assessment, and peer assessment; confidence in self-efficacy and research efficacy; and personal identity essays. Surveys of mentors will identify challenges of senior faculty and ways to incentivize mentoring. For PSIA grantees focused on medical students, a unique metric will be to track those who enter a residency program at another PSIA institution. Pro- grams will evaluate their sustainability, the number of physician-scientists entering and remaining in the career path, and research breakthroughs discovered by MD-only physician-scientists.

\section{Summary and anticipated impact}

Collectively, the PSIA grantee institutions are conducting ten different experiments. Through these efforts, we aim to identify and define key financial and nonfinancial factors and resources needed to create a longitudinally structured and continuous immersive training path to professional independence. Successful elements of the ten PSIA experiments will broaden accessibility of the physician-scientist career path to different clinical disciplines and demographic populations. Establishment of a national network of PSIA programs and formalized relationships with other institutions will continue multidisciplinary support and mentorship for trainees, with each serving as a hub for developing expanded regional collaboratives for physician-scientist training.

Barriers to the successful pursuit of MD-only research careers persist at institutional and national levels and must be addressed in order to develop a critical mass of physician-scientists in the biomedical workforce. The institutional classification of trainees during research years may affect compensation and access to low-cost health insurance. Institutions are increasingly relying on clinical educator faculty, resulting in limited interactions for medical students with physician-scientists. Medical students are encouraged to seek opportunities for higher quantities of publications in order to compete for residency, dissuading their pursuit of discovery science experiences. At the national level, the Accreditation Council for Graduate Medical Education (ACGME) requirements for quality improvement activities during training have limited consideration of laboratory-based research opportunities among residents.

MD research opportunities can be expanded through changes to medical school curricula, summer research training opportunities, and postgraduate physician-scientist training programs that integrate research and clinical training. To support access to these opportunities, it is critical that they not increase the substantial debt burden that faces medical students. Collective investment by federal funding programs, institutions, health systems, and philanthropy will be crucial for successful execution of MD research training programs.

We anticipate the PSIA efforts - combined with necessary policy changes at the institutional and national levels - will effectively lower the hurdles to establishing academic careers and ultimately change the view of medical school, residency, and fellowship to an environment that supports generating physicianscientists throughout the career pipeline. In turn, this investment will increase the number of physician-scientists leading successful research careers.

Address correspondence to: Rasheed A. Gbadegesin, Duke University School of Medicine, Duke Molecular Physiology Institute (DMPI), 300 North Duke Street, Durham, North Carolina 27701, USA. Phone: 919.681.5543; Email: rasheed. gbadegesin@duke.edu.

1. Khot S, et al. The Vietnam war and medical research: untold legacy of the U.S. doctor draft and the NIH "Yellow Berets". Acad Med. 2011 86(4): 502-508.

2. Park BS. The development of the intramural research program at the National Institutes of Health after World War II. Perspect Biol Med. 2003;46(3):383-402.

3. Appointment of associateships for 1967 at the National Institutes of Health. Public Health Rep. 1965; 80(4):370-371. https://www.ncbi.nlm.nih. gov/pmc/articles/PMC1919641/pdf/pubhealthreporig00052-0087.pdf.

4. Harding CV, et al. History and outcomes of 50 years of physician-scientist training in medical scientist training programs. Acad Med. 2017;92(10):1390-1398.

5. National Science Foundation. 2016 Doctorate Recipients from US Universities. https://www. nsf.gov/statistics/2018/nsf18304/static/report/ nsf18304-report.pdf. Accessed March 30, 2021.

6. Association of American Medical Colleges. 2020 FACTS: Enrollment, Graduates, and MD-PhD data. https://www.aamc.org/data-reports/ students-residents/interactive-data/2020facts-enrollment-graduates-and-md-phd-data. Accessed March 30, 2021.

7. Simpson RL,et al. Novel prescriptions from medical schools for physician-scientist training and engagement in the twenty-first century. Acad Med. 2021;96(4):512-515.

8. Duke University School of Medicine. Office of Physician-Scientist Development. https:// medschool.duke.edu/opsd. Accessed March 30, 2021. 
9. Stanford University School of Medicine. Discovery Curriculum. https://med.stanford.edu/md/ discovery-curriculum.html. Accessed March 30, 2021.

10. University of Pittburgh. Physician-Scientist Incubator. https://www.physicianscientist.pitt. edu/. Accessed March 30, 2021.

11. University of Texas Southwestern Medical Center. Training Resident Doctors as Innovators in Science (TARDIS). https://www.utsouthwestern. edu/research/programs/tardis/. Accessed March 30, 2021.

12. Vanderbilt University Medical Center. Vanderbilt SCRIPS: Supporting Careers in Research for Interventional Physicians and Surgeons. https:// www.vumc.org/oor/vanderbilt-scripssupporting-careers-research-interventionalphysicians-and-surgeons. Accessed March 30, 2021.

13. Texas A\&M University. Academy of Physician-Scientists. https://medicine.tamu.edu/aps/ index.html. Accessed March 30, 2021.

14. UCLA David Geffen School of Medicine. BWF Physician-Scientist Training Program. https:// medschool.ucla.edu/research/burroughswellcome-fund-physician-scientist-trainingprogram. Accessed March 30, 2021.

15. University of Chicago. Burroughs Wellcome Fund Early Scientific Training to Prepare for Research Excellence Post-Graduation (BEST-
PREP). https://chess.uchicago.edu/best-prep/. Accessed March 30, 2021.

16. Washington University School of Medicine in St. Louis. Division of Physician-Scientists. https:// physicianscientists.wustl.edu. Accessed March 30, 2021.

17. Weill Cornell Medicine. Burroughs Wellcome Weill Cornell Physician Scientist Program. https://faculty.weill.cornell.edu/facultydevelopment/programs/BWWC. Accessed March 30, 2021.

18. Physician-Scientist Support Foundation. The Medical Student Research Fellowship Program (MSRF). http://www.thepssf.org/msrf/. Accessed March 30, 2021. 